\title{
Un exoesqueleto flexible y textil para asistir la flexión del codo
}

\author{
José Luis Samper-Escudero ${ }^{1,3}$ (joseluis.samper@upm.es), David Cantalejo-Escobar ${ }^{1,3}$, David \\ Tapia $^{3}$, Miguel Ángel Sánchez-Urán ${ }^{1,2}$, Manuel Ferre ${ }^{1,3}$ (m.ferre@upm.es) \\ ${ }^{1}$ CAR UPM-CSIC, Universidad Politécnica de Madrid \\ ${ }^{2}$ ETSIDI, Universidad Politécnica de Madrid \\ ${ }^{3}$ ETSII, Universidad Politécnica de Madrid
} Pont-Esteban ${ }^{1,3}$, Aldo-Francisco Contreras-González ${ }^{1,3}$, Ricardo Ruiz ${ }^{3}$, Fernando Blaya ${ }^{2}$, Santiago

\section{Resumen}

Los exoesqueletos son robots que circunscriben la anatomía humana para asistir su locomoción. Entre las distintas categorías, los exoesqueletos flexibles o exotrajes (del término anglosajón exosuit) se caracterizan por su ligereza y bajas restricciones al movimiento. Los exotrajes emplean principalmente elementos flexibles, como tela, por lo que carecen de una estructura rígida autoportante. Por el contrario, estos robots se apoyan en el sistema musculo-esquelético al que transfieren fuerzas asistenciales. Este artículo describe el sistema para flexar el codo del exoesqueleto LUXBIT. El proyecto LUXBIT busca crear un exoesqueleto portable para asistencia de tareas bimanuales. Para ello emplea un sistema textil de agarre y transferencia de fuerzas al usuario basado en un esquema de polea móvil. El uso de métodos de costura ha proporcionado una solución compacta, fácil de mantener y versátil. Este diseño reduce la presión aplicada sobre la piel. Los últimos apartados muestran los resultados de aplicar el sistema en cuatro sujetos.

Palabras clave: exoesqueleto, exotraje, robot, interación humano-robot.

\section{Introducción}

La irrupción de la robótica en la sociedad contemporánea incluye una amalgama de ejemplos, desde manipuladores robóticos en cadenas de producción a robots sociales, sondas del fondo oceánico o sistemas de entretenimiento. Conforme estos sistemas se integran en la vida cotidiana, el interés por la interacción humano-robot (IHR) se acentúa. Esta excepcional simbiosis ofrece aunar, por una parte, la destreza y capacidad de decisión humana, con la resistencia física, seguridad y precisión de los sistemas robotizados $[5,10,21]$.

Los exoesqueletos son un ejemplo peculiar de cooperación humano-robot (CHR) en el que el robot rodea de forma total o parcial la anatomía humana para asistir su movimiento. Existen múltiples tipos de exoesqueletos que pueden catalogarse siguiendo varios criterios; siendo la distinción estructural entre rígidos y flexibles ampliamente utilizada. Los exoesqueletos rígidos cuentan con una estructura que soporta y dirige el movimiento del usuario. Así,los exoesqueletos rígidos pueden aumentar las capacidades físicas humanas, al elevar grandes cargas [1], por ejemplo, y rehabilitar pacientes con distintos grados de limitación del movimiento $[6,19]$. Aunque la voluminosidad y peso de estos sistemas se ha ido reduciendo a lo largo de su desarrollo, aún existen restricciones al movimiento del usuario [2]. Para prevenir las desalineaciones que provoca la rigidez estructural de estos dispositivos con la anatomía humana, se están investigando nuevas soluciones de actuación y transmisión [20]. Un ejemplo son los mecanismos de rigidez variable (variable stiffness mechanisms) que aproximan de forma aceptable la dinámica articular durante la locomoción [9].

Por otra parte se encuentran los exoesqueletos flexibles, los cuales carecen de una estructura rígida auto-portante. Estos exoesqueletos son livianos y suelen presentarse embebidos en prendas. Los mecanismos de actuación blanda que emplean, como actuadores fluídicos o transmisión por cable, favorecen la compatibilidad con los movimientos humanos [22]. El principio de funcionamiento de estos exotrajes se apoya en la estructura anatómica del usuario a la que transfieren fuerzas que facilitan el movimiento. Así, estos exoesqueletos se destinan principalmente a la reducción del coste metabólico y fatiga asociados a una tarea [13]. Uno de los campos de aplicación más investigados es la asistencia a la marcha. Aunque también existen ejemplos para el tren superior, principalmente focalizados en la muñeca y las manos [7].

La reducción de los problemas musculoesqueléticos se encuentra entre los principales beneficios potenciales de los exotrajes. La Organización Mundial de la Salud (OMS) ha alertado en sucesivas ocasiones del preocupante incremento y falta de soluciones para prevenir y paliar estas afecciones del sistema locomotor[26]. Los distintos organismos de salud erigen las malas posturas y fatiga física como principales causantes o agra- 
vantes del problema, promoviendo así un conjunto de medidas ergonómicas y guías de salud postural [27, 28, 29]. Los exotrajes plantean convertirse en una nueva herramienta con la que afrontar este gran reto social al tener un impacto directo en la fatiga, postura y coste metabólico. A diferencia de los exoesqueletos rígidos que permiten mover cargas medias y grandes, los exoesqueletos flexibles mejoran la postura al reducir ligeramente la fatiga. Aunque sus tasas asistenciales son menores, ofrecen mayor maniobrabilidad y facilitan su uso durante toda la jornada laboral. El objetivo no es por tanto eliminar toda la carga portada, sino aliviar o relajar el estrés muscular evitando una pérdida de tono que pueda derivar en otros problemas musculoesqueléticos. Así, su moderada asistencia y necesidad de cooperar con el usuario permite que se sigan activando y usando los mecanismos de sostén y estabilización del cuerpo. Su compacto y ligero diseño es, a su vez, fácilmente integrable en el equipo y ropa de trabajo [3]. Existen varios ejemplos que prueban los beneficios de estos sistemas al asistir la carga lumbar y posturas incómodas de los trabajadores en distintos sectores industriales [24, 25].

Sin embargo, la estrecha cooperación entre humano y robot de los exotrajes plantea una serie de retos en materia de actuación y sensado. La transferencia de fuerzas requiere del estudio de nuevos sistemas de actuación blanda que permitan imitar la respuesta muscular, como músculos McKibben, SMA o arreglos con cámaras de fluidos, entre otros $[7,23]$. La colocación y adhesión de estos sistemas a la anatomía para asistir el movimiento es otro de los grandes retos para el desarrollo de exoesqueletos flexibles. $\mathrm{Al}$ depender del sistema musculo-esquelético del usuario, los sistemas de actuación deben adaptarse y ser compatibles con el espacio de trabajo del cuerpo humano. En sistemas cableados, por ejemplo, el recorrido de los tendones puede ser alterado por ciertos gestos lo que provoca fallas asistenciales. De forma parecida, los sistemas de sensado también requieren nuevas estrategias con las que monitorizar el estado del robot y del usuario. En este sentido, es de gran interés avanzar en la predicción de la intención de movimiento del usuario y el desarrollo de controladores inteligentes [8, 12].

Este artículo describe el sistema de asistencia a la flexión de codo empleado en el exoesqueleto del proyecto LUXBIT. El mecanismo propone un guiado del tendón que recuerda a un sistema de polea móvil y es compatible con la libre pronosupinación del antebrazo y la rotación glenohumeral. Este sistema, descrito en la sección 2, evita el empleo de correas y cinchas que presionen la piel. Las costuras y tramas del tejido de este método de acoplamiento favorecen la transferencia de fuerzas y adaptación anatómica del diseño. Además, los distintos componentes de la actuación se han integrado en botones para facilitar la puesta en marcha y mantenimiento del dispositivo. El exoesqueleto usa una mochila textil para transportar la electrónica y motores. El diseño ha sido evaluado con cuatro sujetos siguiendo los procedimiento descritos en el apartado 3. Según los resultados del apartado 4, el exoesqueleto es reduce la actividad muscular y mejora la trayectoria de tareas repetitivas, independientemente de la carga transportada. Por último, el apartado 5 recoge las principales conclusiones, limitaciones y propuestas a futuro del estudio.

\section{Descripción del exoesqueleto}

El exoesqueleto del proyecto LUXBIT, en la Fig.1, es capaz de asistir el codo y el hombro en rangos articulares hasta 120 grados. Este modelo usa una mochila textil para facilitar su transporte y uso en distintos entornos, interiores y exteriores. Los tendones usan cables metálicos trenzados con construcciones de 7x7 y 1x19 que ofrecen alta flexibilidad y resistencia a la rotura de hasta $400 \mathrm{~N}$ para el codo. Además, disponen de un recubrimiento de nailon para evitar abrasión y mejorar la durabilidad del sistema. Los motores son actuadores paso a paso Nema-14 de Dings con ensamble lineal. El husillo es de $140 \mathrm{~mm}$ y la tuerca de nailon integra un retén antideslizante. En artículos previos se describe el sistema de control y las distintas estrategias de sensado blando [4, 14, 15, 18].

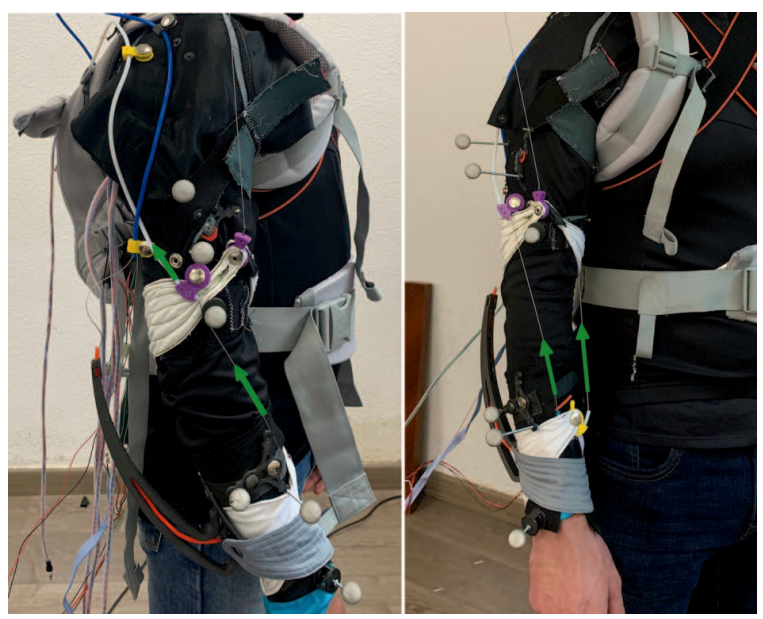

Figura 1: Exoesqueleto flexible LUXBIT. Las flechas verdes indican la fuerza ejercida por el tendón para elevar el codo.

El exotraje usa una chaqueta elástica de ciclismo como tejido base para unificar y estandarizar el diseño. Sobre esta prenda se han cosido los 
parches y componentes textiles que constituyen el exoesqueleto. La trama, forma y composición de estas adiciones ha sido estudiada para absorber las fuerzas de reacción y propagar aquellas que favorecen la adaptación al usuario y el funcionamiento del exoesqueleto. Además, el sistema cableado es desmontable y ajustable a distintas complexiones anatómicas mediante el uso de botones.

La mochila es un producto comercial con certificación ergonómica para transportar cargas de hasta $20 \mathrm{~kg}$. El diseño original se ha modificado mediante la incorporación de una capa en la espalda de espuma mallada, dos bandas elásticas que sujetan la mochila al tronco y la reubicación del relleno en las asas. Los componentes de la actuación se fijan en la mochila con botones perforados en los que se aprieta un perno. El peso total de la mochila para asistir sendos codos en flexión es de 700 gramos, mientras que en los brazos recaen 265 gr.

En cuanto a la asistencia, el motor es capaz de proporcionar fuerzas de $40 \mathrm{~N}$ a $110 \mathrm{~N}$, dependiendo de la velocidad. En este caso se ha seleccionado una velocidad media-lenta que permite flexar el codo en 4 segundos. Esta limitación previene sobrecargas de los tendones y es suficiente para evaluar gestos de la jornada laboral. Además, el diseño se ha calculado para que las piezas de la actuación y el anclaje de los cables se suelten en caso de superar fuerzas superiores a $200 \mathrm{~N}$.

\subsection{Interfaz de acoplamiento textil}

Para un sistema cableado vestible, el método con que se transfiere la fuerza de movilización al usuario determina la comodidad y viabilidad del exoesqueleto. En lugar de usar cinchas o telas compresivas de alta resistencia a la fatiga, como el neopreno, se propone un diseño transpirable y autoajustable que sólo aplica presión a la piel durante la asistencia. El método propuesto, en la Fig. [?], está completamente basado en textiles. La abrazadera textil diseñada tiene forma de $v \mathrm{y}$ está constituida por dos capas de tela ProCool@ Athletic Interlock Silver. Las capas están rotadas entre sí para conseguir que la urdimbre, la dirección que aporta rigidez, vaya del eje de simetría de la abrazadera a uno de los extremos.

En cada extremo o punta de la abrazadera se ha colocado la cara de un botón, de forma que puedan abotonarse entre sí formando un brazalete. Las costuras en la abrazadera textil se han realizado para que éstas transfieran el tirón que ejerce el cable sobre el botón al eje de simetría de la misma. De esta forma, todas las costuras parten del centro del botón y se dirigen a puntos del eje de simetría equidistantes entre sí. Así, el principio de fun- cionamiento de esta propuesta de acoplamiento consiste transferir la tensión del tendón a un botón que a su vez distribuye la fuerza entre varias costuras planas. Estas costuras propagan la fuerza desde la parte anterior del antebrazo, donde está el botón, al eje de simetría que genera una fuerza efectiva distribuida por la parte posterior del antebrazo, la cual fuerza la flexión del codo. Dos aspectos destacan del diseño: por un lado se tiene que el tirón puntual del tendón se transforma en una fuerza distribuida sobre un área; por otro, el tejido sólo aplica presión cuándo el cable está en tensión (ver Fig. 2). Por lo tanto, el diseño reduce la compresión de la piel al quedar holgado cuando el cable no está tenso. Esta solución pretende favorece la comodidad y usabilidad del dispositivo, especialmente en jornadas largas.

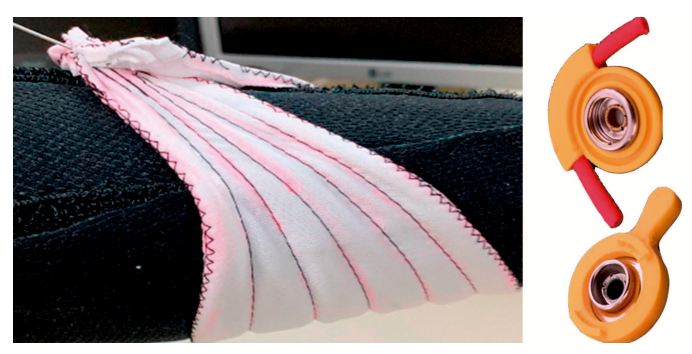

Figura 2: La imagen de la izquierda muestra la propagación de fuerzas por las costuras al tensar el cable. Los componentes de la derecha son la guía curva integrada en un botón que une los dos extremos de la abrazadera textil.

La curvatura de las costuras y la forma del acople textil se han obtenido siguiendo un procedimiento en software 3D-CAD ya descrito en otras publicaciones $[16,17]$. Finalmente, el acople textil se une con una costura recta en su eje de simetría a un brazalete elástico de ProCool@ Stretch-FIT DriQWick $^{T M}$ que tiene cierre con velcro. Nótese que todos los tejidos son transpirables, antibacterianos $\mathrm{y}$ finos.

\subsubsection{Mecanismo para flexar el codo}

El exoesqueleto asiste la flexión del codo al colocar un punto de pivote o apoyo en el brazo y la abrazadera textil en el antebrazo. Una explicación simplificada es que el tendón tira del acople textil apoyándose para ello en el pivote del brazo, lo que genera un par de giro en el codo. El sistema propuesto, no obstante, plantea dos modificaciones en el recorrido del cable para mejorar la compatibilidad anatómica de la asistencia.

En la Fig. 3, el cable está anclado a una hebilla sobre el tríceps. De ahí, pasa por un punto de pivote en la cara interna del bíceps y va a la abrazadera textil del antebrazo. En el botón de 
esta abrazadera se ha integrado una guía circular con una funda de bajo rozamiento que favorece el deslizamiento del cable en ella, como muestra la Fig. 2. El cable es así redirigido por la guía a la parte externa del brazo donde interactúa con un segundo punto de pivote, simétrico al de la cara interna, que inserta el tendón en una funda que lo guía hasta el motor en la mochila.

El guiado del cable a través de las caras laterales del brazo reduce las perturbaciones por las contracciones del bíceps. Además, la simetría del montaje y la guía circular permiten que las longitudes se reajusten para balancear las fuerzas a cada lado del botón. Así, el usuario puede pronosupinar el antebrazo y realizar rotaciones glenohumerales a voluntad sin alterar el funcionamiento del mecanismo de flexión del codo.

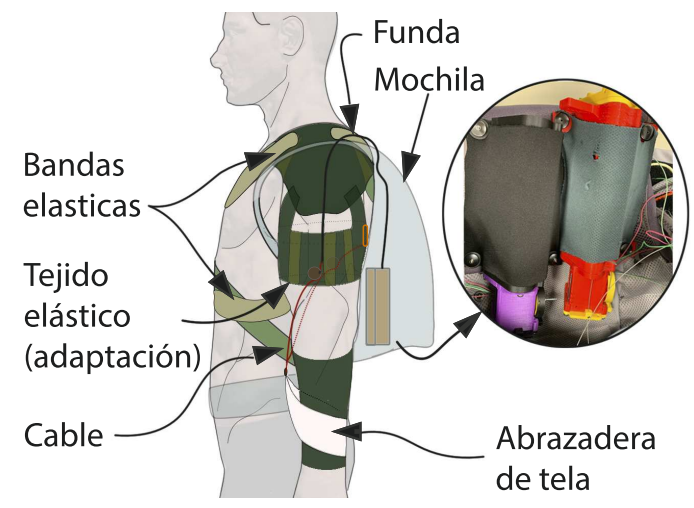

Figura 3: Esquema del cableado para el codo con vista ampliada de la fijación de los motores lineales que actúan cada brazo.

La integración de los componentes de actuación en botones, como las guías y los pivotes, facilita el montaje y reduce la voluminosidad del exoesqueleto. Las guías de las fundas y los pivotes pueden además anclarse en distintos puntos para adaptar el diseño a la constitución anatómica de cada usuario. Para la colocación de los elementos se ha establecido un acuerdo entre el par de giro generado en el codo, las fuerzas de reacción sobre los botones y la composición anatómica del brazo [17]. Sin ahondar en los detalles, las zonas próximas a las articulaciones tienen mayor concentración de tejidos densos o duros (tendones, hueso, cartílago, etc); mientras que las zonas centrales concentran mayormente tejidos blandos como músculos. Además, hay una correlación entre la altura del usuario y el grosor medio del brazo. De este modo, la abrazadera se coloca entre la muñeca y la zona media de los flexores del antebrazo; y el brazal con los pivotes bajo la línea media del bíceps.

\section{Métodos experimentales}

El exoesqueleto se ha evaluado en cuatro sujetos sanos, debidamente informado según las normas de la Universidad Politécnica de Madrid. En ellos, los sujetos, con edades comprendidas entre los 22 y 32 años, tenían que ponerse el exoesqueleto y realizar cinco repeticiones de flexión de codo. La actividad muscular del bíceps y la trayectoria del brazo fueron monitorizadas durante todo el experimento con Optitrack y un sensor electromiográfico MyoWare. Nueve marcadores de Optitrack se pusieron sobre el usuario según el protocolo Biomech [11], aunque fueron necesarias algunas modificaciones para prevenir oclusiones con las cámaras y espacio de experimentación disponible. Las guías para selección y uso de electrodos SENIAM fueron aplicadas para la electromiografía superficial (sEMG).

Los ejercicios realizados consistían en repetir cincos flexiones de codo hasta noventa grados. El número de repeticiones se ha seleccionado evaluando el número de repeticiones con peso a partir del cual los sujetos mostraban fatiga muscular y saturación del sensor. Se decidió que evitar la saturación muscular durante las pruebas favorecía la evaluación, especialmente en la trayectoria. El patrón de movimiento durante las pruebas era: flexar el codo en cuatro segundos, mantener la flexión de 90 grados durante dos segundos, bajar el brazo en cuatro segundos y, finalmente, mantener la posición inicial durante dos segundos. Los participantes hicieron el experimento primero sin asistencia y después con ayuda del exoesqueleto; descansado hasta cinco minutos entre ejercicios para prevenir fatiga previa. Después, se les entregó una pesa de un kilogramo y tuvieron que repetir el experimento, sin y con asistencia.

Para la adquisición y procesamiento de los datos se usó una tarjeta NVidia Jetson Nano y una placas de Texas Instrument LaunchXL F28379D. La señal sEMG adquirida en el Biceps con el sensor sEMG MyoWare fue normalizada para cada sujeto usando los resultados de una prueba de esfuerzo máximo realizada al finalizar los experimentos. De forma similar, Optitrack entrega la posición y orientación de cada marcador en cuaternios, debiendo aplicándose posteriormente una relación de cuerpos rígidos a los marcadores sobre el mismo hueso o segmento corporal con interpoladores cúbicos. El procesamiento se llevo a cabo en Matlab y Motive. El software Motive se empleó para revisar y corregir manualmente las etiquetas de los marcadores no anotados correctamente durante las pruebas y aplicar un interpolador cúbico. Además, éste software integra una herramienta con la que establecer y generar las 
trayectorias de los cuerpos rígidos a partir de los marcadores colocados sobre él. Para ello, se han de asignar manualmente los marcadores al cuerpo rígido una vez corregidas todas las etiquetas y resueltas las oclusiones.

\section{Resultados}

Para analizar los resultados se ha obtenido la asistencia y mejora relativa cuando se usa el exoesqueleto, respecto al caso no asistido para cada usuario. La tabla 1 recoge la tendencia central de la asistencia relativa obtenida para cada sujeto. Así, las dos primeras columnas denotan la magnitud relativa en que la actividad muscular ha mejorado, esto es, el exoesqueleto reduce la actividad muscular (valor positivo); o empeorado (valor negativo). Seguidamente, las columnas 3 y 4 indican si el exoesqueleto mejora o empeora el ángulo máximo alcanzado así como la variabilidad entre los picos de las cinco repeticiones. Por último, las dos últimas columnas da una medida de el tiempo que duró el ejercicio y que los sujetos permanecieron en la postura de máxima fatiga (flexión a 90 grados). Para el cálculo de estos valores, se emplean las relaciones $\left(v_{n} o\right.$ Asistido $-v_{A}$ sistido $) / v_{n} o$ Asistido y $\left(v_{A}\right.$ sistido $-v_{n} o$ Asistido $) / v_{n} o$ Asistido, de forma que un valor positivo indique un beneficio positivo al usar el exoesqueleto.

Los resultados muestran que el exoesqueleto reduce la actividad muscular en una actividad repetitiva de corta duración (un minuto) en una media de $6.25 \%$ y $5.86 \%$ para los cuatro sujetos. Este beneficio es significativo cuando se valora el incremento de tiempo que los sujetos han permanecido manteniendo una posición de alto estrés muscular. Según la tabla 1, el brazo se mantuvo flexionado en la posición máxima un $57.40 \%$ y $57.45 \%$ más cuando el exoesqueleto estaba activo. Es así llamativo que el exotraje reduzca la señal electromiográfica a pesar del inherente incremento del estrés muscular que ello conlleva.

Hay indicios además que apuntan que el exoesqueleto es capaz de proporcionar una tasa asistencial mayor, tal y como ilustra la imagen 4 . Estas gráficas muestran los resultados para un sujeto mientras realizada los ejercicios, con y sin peso. Los dos observaciones más llamativas son el ángulo máximo alcanzado y la mejora de la trayectoria. Durante las pruebas, el sujeto sobrepasó la flexión de noventa grados; por lo que el exoesqueleto dejó de asistirle en las bandas sombreadas en rojo. Esto conllevó un incremento de la actividad muscular, como muestran los picos marcados en morado. Es interesante observar que estos picos tienen menor magnitud en la situación con carga ya que el par-

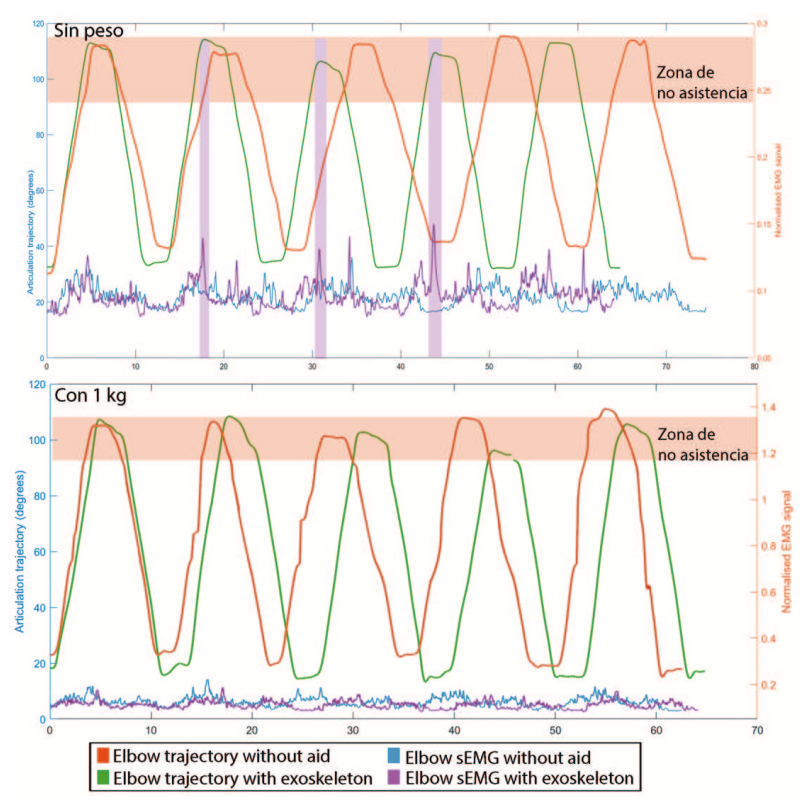

Figura 4: Resultados de trayectoria y actividad muscular para un sujeto. La banda roja muestra la zona que no era asistida por el exoesqueleto. Los picos se correspondan con la máxima flexión alcanza como ilustran las bandas púrpura.

ticipante redujo la banda no asistida. El caso con peso evidencia también que el exoesqueleto puede reducir significativamente la actividad muscular.

Las gráficas también muestran el beneficio del exoesqueleto para la ejecución de un gesto. Específicamente, el sujeto mejora la linearidad del movimiento, el ángulo máximo alcanzado, el tiempo que mantuvo la flexión máxima y el rango articular. No obstante también demuestra que al entrar en un banda no asistida, el usuario tiende a reducir progresivamente el ángulo de flexión que mantiene en el tope del gesto. La entrada en la banda sin asistencia también provoca una mayor variabilidad en el ángulo pico. En ambos casos, con y sin carga, la respuesta del sujeto es parecida: en las primeras repeticiones alcanza un ángulo de flexión sobre los cien grados; después, el cansancio provoca una reducción de éste aproximándose al límite de asistencia programando en el exoesqueleto, noventa grados; finalmente, al ser las últimas repeticiones, el participante trata de recobrar el ángulo inicial. Este patrón de movimiento se observa también en los casos no asistidos, especialmente en el que se porta la carga.

En términos genéricos, el dispositivo reduce la variabilidad del gesto notablamente, en un $69.84 \%$ y $23.37 \%$. La mayor reducción del caso sin peso puede achacarse al impacto de la carga en la propiocepción humana al realizar el gesto. Por otra 
Tabla 1: Resultados de asistencia relativa, en porcentaje.

\begin{tabular}{|c|c|c|c|c|c|c|}
\hline Prueba & $\begin{array}{c}\text { sEMG } \\
\text { medio }\end{array}$ & $\begin{array}{c}\text { sEMG } \\
\text { max. }\end{array}$ & $\begin{array}{c}\text { Ang. } \\
\text { max. }\end{array}$ & $\begin{array}{c}\text { Std. Ang. } \\
\text { max. }\end{array}$ & Duración & $\begin{array}{c}\text { Tiempo } \\
\text { flex. }\end{array}$ \\
\hline Codo & 6.25 & -2.94 & 4.27 & 69.84 & 18.66 & 57.40 \\
Codo $1 \mathrm{~kg}$ & 5.86 & 2.01 & -3.81 & 23.37 & 13.77 & 57.45 \\
\hline
\end{tabular}

Todos los valores son relativos al valor del usuario sin asistencia de cada caso. Las abreviaciones son: Max., máximo; Std., desviación típica; Ang., ángulo de trayectoria; Flex., flexión; Duración, es la duración de la prueba, y Tiempo flex., el porcentaje de tiempo que los sujetos aguantaron la flexión de 90 grados. Un resultado positivo indica el beneficio del exoesqueleto para ese parámetro en cuestión.

parte, el exoesqueleto ha beneficiado el ángulo máximo medio alcanzado por los individuos en el caso sin peso; por el contrario, éste se ha reducido en un $3.81 \%$ al portar una carga. Al revisar los datos, se observa que sin el exoesqueleto los sujetos alcanzaron un ángulo medio máximo de $86.76^{\mathrm{O}}$ al portar la mancuerna de un kilogramo. Con el exoesqueleto, sin embargo, el ángulo fue de $83.32^{\mathrm{o}}$. Esto denota una falta en el funcionamiento del exoesqueleto siendo dos explicaciones las de mayor probabilidad: por un lado, se tiene que el exoesqueleto no mide directamente la presión o fuerza aplicada por la abrazadera textil. Por lo tanto, el incremento de cualquier coeficiente de fricción puede inducir a una falta de asistencia. La fijación de los marcadores de Optitrack requirió colocar tornillos de cinco centímetros cerca de algunos tendones, haciendo que éstos rozasen entre sí durante el movimiento. Por otro lado, se tiene que la altura de los sujetos seleccionados, de $1.81 \mathrm{~m} \pm 0.082 \mathrm{~m}$, requirió en algunos casos emplear el máximo recorrido del motor lineal pudiendo quedar los últimos grados sin asistencia por el volumen de la tuerca y factor de seguridad del control que reduce el largo efectivo del husillo.

Recapitulando, el exoesqueleto demuestra capacidad para mejorar la ejecución de gestos repetitivos y mantener posturas incómodas. Un resultado interesante es el incremento del tiempo que el usuario puede permanecer en una postura de alto estrés muscular sin afectar la actividad muscular. Los resultados han probado que los sujetos mantuvieron el peso durante dos segundos mostrando una actividad muscular inferior a la que corresponde a un gesto que dure un $57.40 \%$ menos (0.86 segundos). Se ha demostrado además que el usuario puede realizar movimientos libremente y sobrepasar la posición asistida por el exoesqueleto. $\mathrm{Al}$ descansar también puede reacomodar la articulación o modificar el ángulo articular libremente. Por último, se ha detectado un punto de mejora que puede deberse a fricciones parásitas o limitaciones del motor. En cualquier caso, son de menor importancia y serán resueltas en trabajos futuros.

\section{Conclusiones}

El exoesqueleto descrito en este artículo es un diseño basado en textiles para asistir la flexión de codo. El sistema es portátil mediante el uso de una mochila textil ergonómica para la espalda que ha sido modificada en el laboratorio para mejorar algunos aspectos como la sujeción al tronco o un acolchado en la espalda. La abrazadera textil para transferencia de fuerzas ha demostrado su funcionalidad y aplicabilidad dado su reducido peso, transpirabilidad y comodidad. El sistema de guiado simétrico del cable es capaz de reducir la actividad muscular asociada a una tarea y mejorar la trayectoria del usuario. La solución presentada no es restrictiva y permite al usuario combinar el resto de movimientos del brazo que no son asistidos, lo que favorece la aplicación del sistema a entornos laborales y de la vida cotidiana. La integración de la actuación en botones y el estudio de la composición textil ha demostrado favorecer la compactación y ligereza del dispositivo. Además, el diseño es higiénico y puede lavarse y desinfectarse cómodamente. Se debe notar que el diseño ha sido puesto durante más de treinta horas y no ha requerido mantenimiento o reparación de ningún componente.

En términos asistenciales, el exoesqueleto ha demostrado mejorar el tiempo que un usuario puede mantener una carga en una postura incomoda. Sorprendentemente, el dispositivo ha conseguido que este incremento no conlleve una mayor fatiga muscular. El sistema también mejora la repetibilidad y linearidad del gesto. No obstante, la evaluación tiene dos limitaciones: el tamaño de la muestra y duración. Al contar con sujetos sanos y focalizar aplicaciones a la vida cotidiana es conveniente realizar próximas evaluaciones con un mayor tamaño muestreal con potencia estadística significativa. Además, se debe notar que el experimento ha permitido evaluar los beneficios del dispositivo en una situación especifica y poco favorable en la que el sujeto no alcanza la fatiga muscular. Así, sería interesante aumentar las repeti- 
ciones y duración del ejercicio a fin de evaluar la hipótesis de mejora del beneficio con el tiempo. En este mismo contexto, convendría incluir ejercicios multitareas que requieran al usuario realizar distintas poses y gestos individuales ya que es en estos ejercicios de escasa repetibilidad donde los exoesqueletos flexibles como el presentado destacan frente a otras tendencias más restrictivas y rígidas. Por último, entre los trabajos futuros destaca la mejora de la realimentación de fuerzas, el alto-nivel del sistema de control y el aumento del husillo efectivo o ratio cable desplazado - husillo recorrido.

\section{Agradecimientos}

Los trabajos de este artículo han sido parcialmente financiados por el Ministerio de Ciencia e Innovación, bajo el proyecto LUXBIT (RTI2018094346-BI00).

\section{English summary}

\section{A portable flexible exoskeleton to as- sist elbow flexion}

\begin{abstract}
Exoskeletons are robots that surround the human anatomy to assist locomotion. Flexible exoskeletons, so-called exosuits, use soft materials and actuation methods to deliver a lightweight and unobstrusive construction. Their lack of a rigid self-supportive structure requires them to support their functioning on the users' anatomy. This paper covers the mechanism to flex the elbow used by the flexible exoskeleton LUXBIT. The LUXBIT project aims to attain a flexible portable exoskeleton to assist the arms in bimanual tasks. The exoskeleton has a textile coupling that combines fabrics' stacking and sewing patterns to transfer mobilising forces to the user. The actuation's compactness has been achieved by applying tailoring methods. The resulting design is versatile, easy to maintain, and does not apply pressure to the skin constantly. The last sections evaluate the exoskeleton in four healthy subjects.
\end{abstract}

\section{Keywords:Exoskeleton, exosuit, robot, human-robot interaction.}

\section{Referencias}

[1] Babic, Jan, et al. Power-augmentation control approach for arm exoskeleton based on human muscular manipulability. En 2017 IEEE International Conference on Robotics and Automation (ICRA). IEEE, 2017. p. 5929-5934.

[2] Chen, Bing, et al. Recent developments and challenges of lower extremity exoskeletons. Journal of Orthopaedic Translation, 2016, vol. 5, p. 26-37.

[3] Colombini, Daniela; OCCHIPINTI, Enrico. Preventing upper limb work-related musculoskeletal disorders (UL-WMSDS): New approaches in job (re) design and current trends in standardization. Applied ergonomics, 2006, vol. 37, no 4, p. 441-450.

[4] Contreras-González, Aldo F., et al. SoftWearable Device for the Estimation of Shoulder Orientation and Gesture. En International Conference on Human Haptic Sensing and Touch Enabled Computer Applications. Springer, Cham, 2020. p. 371-379.

[5] Dahmen, Christian, et al. Approach of optimized planning process for exoskeleton centered workplace design. Procedia CIRP, 2018, vol. 72 , p. 1277-1282.

[6] Ganguly, Amartya, et al. Wearable pediatric gait exoskeleton-a feasibility study. En 2018 IEEE/RSJ International Conference on Intelligent Robots and Systems (IROS). IEEE, 2018. p. 4667-4672.

[7] Hope, James; MCDAID, Andrew. Development of wearable wrist and forearm exoskeleton with shape memory alloy actuators. Journal of Intelligent Robotic Systems, 2017, vol. 86, no 3-4, p. 397.

[8] Lanotte, Francesco, et al. A low-back exoskeleton can reduce the erector spinae muscles activity during freestyle symmetrical load lifting tasks. En 2018 7th IEEE International Conference on Biomedical Robotics and Biomechatronics (Biorob). IEEE, 2018. p. 701-706.

[9] Li, Zhongyi, et al. Design, modeling and testing of a compact variable stiffness mechanism for exoskeletons. Mechanism and Machine Theory, 2020, vol. 151, p. 103905. 
[10] Michalos, George, et al. Design considerations for safe human-robot collaborative workplaces. Procedia CIrP, 2015, vol. 37, p. 248-253.

[11] [Online] Optitrack: Biomech."https://v20.wiki.optitrack.com/ index.php?title=Biomech_(57)". Accedido: 18.06.2021.

[12] Pacifico, Ilaria, et al. An experimental evaluation of the proto-mate: a novel ergonomic upper-limb exoskeleton to reduce workers' physical strain. IEEE Robotics Automation Magazine, 2020, vol. 27, no 1, p. 54-65.

[13] Panizzolo, Fausto A., et al. A biologicallyinspired multi-joint soft exosuit that can reduce the energy cost of loaded walking. Journal of neuroengineering and rehabilitation, 2016, vol. 13, no 1, p. 1-14.

[14] Pont, David, et al. Exoflex: An upper-limb cable-driven exosuit. En Iberian Robotics conference. Springer, Cham, 2019. p. 417-428.

[15] Pont-Esteban, D., et al. Validation of An Elbow Position Super-Twisting Sliding-Mode Controller for Upper-Limb Exosuit Using A Soft Position Sensor. En Journal of Physics: Conference Series. IOP Publishing, 2021. p. 012074.

[16] Samper-Escudero, José Luis, et al. A CableDriven Exosuit for Upper Limb Flexion Based on Fibres Compliance. IEEE Access, 2020, vol. 8, p. 153297-153310.

[17] Samper-Escudero, José Luis, et al. Assessment of an upper limb exosuit with textile coupling. En 2020 IEEE International Conference on Human-Machine Systems (ICHMS). IEEE, 2020. p. 1-6.

[18] Samper-Escudero, J. Luis, et al. Efficient multiaxial shoulder-motion tracking based on flexible resistive sensors applied to exosuits. Soft robotics, 2020, vol. 7, no 3, p. 370-385.

[19] Sanz-Merodio, Daniel, et al. EXOtrainer project clinical evaluation of gait training with exoskeleton in children with spinal muscular atrophy. En Advances in Robotics Research: From Lab to Market. Springer, Cham, 2020. p. 211-227.

[20] Rathore, Ashish, et al. Quantifying the human-robot interaction forces between a lower limb exoskeleton and healthy users. En 2016 38th Annual International Conference of the IEEE Engineering in Medicine and Biology Society (EMBC). IEEE, 2016. p. 586-589.
[21] Rupal, Baltej Singh, et al. Lower-limb exoskeletons: Research trends and regulatory guidelines in medical and non-medical applications. International Journal of Advanced Robotic Systems, 2017, vol. 14, no 6, p. 1729881417743554.

[22] Thalman, Carly M., et al. A novel soft elbow exosuit to supplement bicep lifting capacity. En 2018 IEEE/RSJ International Conference on Intelligent Robots and Systems (IROS). IEEE, 2018. p. 6965-6971.

[23] Tiseni, Luca, et al. On the edge between soft and rigid: an assistive shoulder exoskeleton with hyper-redundant kinematics. En 2019 IEEE 16th International Conference on Rehabilitation Robotics (ICORR). IEEE, 2019. p. 618-624.

[24] [Online] Verve Motion. "https://vervemotion.com/technology". Accedido: 08.07.2021.

[25] Wolff, Jamie, et al. A survey of stakeholder perspectives on exoskeleton technology. Journal of neuroengineering and rehabilitation, 2014, vol. 11, no 1, p. 1-10.

[26] [Online] World Health Organization. "https://www.who.int/news-room/factsheets/detail/musculoskeletal-conditions". Accedido: 18.06.2021.

[27] World Health Organization (WHO) et al. "International minimum requirements for health protection in the workplace". World Health Organization 2017.

[28] World Health Organization (WHO) et al. "Occupational safety and health in public health emergencies: a manual for protecting health workers and responders". World Health Organization 2018.

[29] WHO Scientific Group on the Burden of Musculoskeletal Conditions at the Start of the New Millennium. "The burden of musculoskeletal conditions at the start of the new millenium : report of a WHO scientific groups". WHO technical report series; 919. 2003, pp 218.

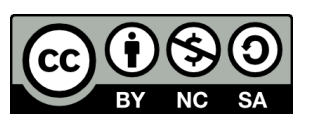
(C) 2021 by the authors. Submitted for possible open access publication under the terms and conditions of the Creative Commons Attribution CC BY-NC-SA 4.0 license (https://creativecommons.org/licenses/by-ncsa/4.0/deed.es). 